\title{
Inter-site: a new tool for the simulation of spatially realistic population dynamics
}

\author{
F. Oliver Gathmann *, D. Dudley Williams \\ University of Toronto, Surface and Groundwater Ecology Research Group, Division of Life Sciences, 1265 Military Trail, \\ M1C 1A4 Scarborough, Ontario, Canada
}

\begin{abstract}
The inter-site simulator, a new tool for simulating spatially realistic population dynamics, is presented. Based on a discrete-event simulation system in an object-oriented programming environment, this simulator aims to offer a general and user-extensible framework for the simulation of spatially structured populations, including metapopulation systems. The simulator is implemented in Python, a versatile member of a recent class of high-level scripting languages, and uses the Tcl/Tk/Tix libraries as GUI front-end. This paper outlines the theoretical concepts underlying the simulator, describes its architecture, and demonstrates the use of the system by re-constructing a classical, state-variable oriented model drawn from literature that investigates the relationship of meta-population persistence with spatial patch arrangement. The paper concludes with a brief discussion of the benefits and drawbacks of the simulation approach to ecological modeling, and with contemplating the future potential of the inter-site system. (C) 1998 Elsevier Science B.V. All rights reserved.
\end{abstract}

Keywords: Spatially realistic population modeling; Metapopulation; Individual-based modeling; Discrete event simulation; Python

\section{Introduction}

Incorporating the spatial dimension into theoretical models of population dynamics has been one of the main focuses of theoretical ecology for more than a decade (Chesson, 1981; Kareiva, 1990; Hastings, 1990). The most visible result of these efforts is perhaps the establishment of

* Corresponding author. Tel.: + 1416 2877420; fax: + 1 416 2877423; e-mail: gathmann@scar.utoronto.ca metapopulation biology as a field in its own right (see overviews in Gilpin and Hanski, 1991; Hanski and Gilpin, 1997). Earlier meta-population models were simple patch-occupancy models that lacked spatial structure, a reflection on the original definition of a metapopulation as 'population of populations' given by Levins (1970), which clearly did not envision explicit spatial relationships among sub-populations. In contrast, modern definitions of the term emphasize the spatial isolation of the sub-populations and view meta- 
populations in the traditional sense as a subset of the more general class of patchy populations (Harrison, 1994). Also, processes of dispersal among sub-populations, or 'patches', have been explicitly integrated into the metapopulation concept, again emphasizing its spatial connotations. Following this conceptual shift, the majority of recent metapopulation models are spatially structured, typically devised in one of the following two ways (Hanski and Simberloff, 1997): (1) as spatially explicit models, representing space as a regular grid and permitting only local interactions among neighboring cells (cellular automata: Ermentrout and Edelstein-Keshet, 1993; coupled map lattices: Hassell et al., 1991; Kaneko, 1992); or (2) as spatially realistic models, incorporating the actual geometry of the patch network and possibly patch size (e.g. Hanski, 1994; Gustafson and Gardner, 1996).

One of the driving forces behind the introduction of spatially extended meta-population models was the discovery of their value as a conceptual tool in conservation biology (Reich and Grimm, 1996; Hanski and Simberloff, 1997). Since the late 1980's, conservation biologists have been faced with a pressing need for scientifically guided habitat management plans for a rapidly growing number of species that are driven to the brink of extinction as a consequence of the ongoing massive habitat destruction and fragmentation by man (Morris, 1992). As populations of endangered species are typically highly patchy and depend on successful dispersal among the remaining isolated habitat patches for long-term persistence, spatially structured metapopulation models represent an ideal framework for analyzing their persistence times under different environmental scenarios. The habitat management plans for the spotted owl Stix occidentalis (e.g. McKelvey et al., 1993; Lahaye et al., 1994; but see Harrison et al., 1993 for a critical review) and for Bachman's sparrow Aimophila aestivalis (Liu et al., 1995) are probably the most prominent examples in this important and still expanding field of applied meta-population biology.

The application of advanced theoretical models of spatially structured populations was facilitated by the development and use of new modeling techniques. One of the most influential innovations in this field was the introduction of individual-based models (also known as i-state configuration models; Caswell and John, 1992) into ecology by Huston et al. (1988). Contrary to the classical state-variable models, which lump individuals of the same state together, individualbased models create and track each and every individual separately and assemble global, population-level information ('p-state' information sensu Metz and Diekmann, 1986) from their local (inter-)actions. This technique is, even for simple population systems, computationally very demanding and the recent popularity of individualbased models among ecologists is at least partly a consequence of the ongoing exponential growth in computer hardware performance (Bullock, 1994). A vast number of individual-based models capturing the dynamics of very different ecological systems has been published in recent years (e.g. DeAngelis et al., 1984; MacKay, 1992; Stelter et al., 1994; Blanche et al., 1996; Humphries et al., 1996; Jaworska et al., 1996; Rose et al., 1996; Hill et al., 1997).

While ecological modelers are very concerned about the epistemological implications of this new modeling style-for instance, how it trades off the complementary goals of 'generality' and 'testability' in model building (Murdoch et al., 1992; Judson, 1994), or whether it really amounts to a qualitatively new approach to ecological modeling, as stated by Uchmanski and Grimm (1996) - little attention has been paid to the issues involved in implementing individual-based models. Frequently, the soft- and hardware used to implement a particular model are barely mentioned in the model description section, probably because it is generally assumed that different implementations of the same mathematical model should essentially be equivalent (Gross et al., 1992; see e.g. Baveco and Lingeman, 1992; Haefner, 1992; Palmer, 1992; Lhotka, 1994; Ferreira, 1995; Laval, 1995; Mooij and Boersma, 1996 for exceptions). However, in light of the astonishing speed at which software technology has evolved over the course of the last two decades, this assumption has to be critically reviewed. Although we should be safe in assuming 
that different implementations of the same model yield (nearly) identical numerical results, a whole suite of implementation-related issues will decisively affect the usefulness of any modeling program as well as the time spent on its development (cf. Maxwell and Costanza, 1997; see also section 2).

This paper pursues two goals: firstly, it introduces the inter-site simulator as a new, highly flexible and easy-to-use tool for the simulation of spatially realistic population dynamics. This is done by outlining its architecture and by presenting a sample application. Secondly, it attempts to vitalize the discussion about the technical issues involved in implementing simulation models of ecological systems by presenting some of the computer sciences methodology and terminology used for developing the inter-site system in more detail.

\section{Implementation of the inter-site simulator}

In this section, we first guide the reader through the series of design decisions that had to be made during development of the inter-site simulator: adopting an adequate programming paradigm (2.1), choosing a programming language (2.2) and selecting a simulation technique (2.3). We then continue with outlining the architecture of the simulator and its general usage (2.4).

\subsection{Programming paradigm}

When challenged with the daunting task of imagining a metapopulation system in their mind, ecologists would most likely envision a set of more or less well-defined objects-like individuals' and 'patches' - that have certain properties and interact in various ways - rather than a network of disembodied processes determining the values of some result variables. This is roughly why the transition from procedural programming to object-oriented programming (OOP) during the 1980's had such a profound impact on the way models are built in engineering and science, including ecology. We had no reason not to follow this trend and decided to implement the inter-site simulator using the OOP paradigm. Apart from classical textbooks (e.g. Booch, 1994; Graham, 1994), a number of introductions to OOP for ecologists are already available (Maley and Caswell, 1993; Ferreira, 1995); hence we restrict ourselves to repeating only the basic concepts necessary to understand the simulator architecture here.

In OOP, functions and data-corresponding to processes and variables of the real world system-are unified in a single data structure called 'object'. To distinguish object functions and data from their traditional counterparts, they are called object 'methods' and 'attributes', respectively (the latter are also known as 'data members'). The method and attribute definitions of an object are contained in its 'class', which serves as a blueprint for the generation of an arbitrary number of object 'instances' during runtime. A mechanism called 'inheritance' allows classes to pass on their methods and attributes to other classes, which are then called 'sub-classes' of the original class. Sub-classes can either define additional methods and attributes, or re-define the ones they inherited ('polymorphism').

Within the framework just presented, it is straightforward to map almost arbitrary realworld systems directly into a corresponding system of object classes. Using our introductory example, a metapopulation system could be represented by the classes 'Area' (the spatial matrix), 'Patch' (local patches), and 'Population' (local populations). Each class would define methods implementing its own dynamic changes and their effects on other objects - for instance, the Patch class could define an 'environmental catastrophe' method eradicating the population object associated with it and the Population class could feature a 'migration' method implementing dispersal to other population objects. However, having defined the objects and their individual dynamics in correspondence to a real-world system still leaves us with the task of organizing the dynamics of the whole system, a question we address in the section on simulation techniques (2.3). 


\subsection{Programming language and environment}

A modern language used as a simulation environment should offer more than simple numbercrunching routines. Object orientation (see section 2.1), optimized high-level data types such as lists, binary trees and dictionaries, a powerful random number generator capable of delivering independent random number streams from various probability distributions, and powerful routines for the manipulation of homogeneous numeric arrays are some of the most desirable properties. A clear syntax is an enormous time-saver, both during development of new and during extension of existing code. Other valuable assets are seamless integration with a graphical user interface (GUI), including routines for data plotting, interfaces to existing database standards, and, of increasing importance, a library of internet services.

Also, it was felt that a customizable, userfriendly simulation system should be written in a language that was both easy to comprehend and accessible to everybody. Python, a simple yet strikingly versatile and powerful scripting language, turned out to be the first choice for the implementation of the inter-site simulator. Python is fully object-oriented, offers high-level programming constructs, has been ported to all major computing platforms and is freely available in source form on the internet. As an interpreted language, Python is ideally suited for the incorporation of user-defined code, which instantly can be tested for errors without recompilation.

The current implementation of the inter-site simulator was developed using Python version 1.5 (van Rossum, 1997) together with the Numeric Python package for fast processing of homogenous numeric arrays (Hugunin, 1997) and the 'Universal Random Number Generator' package for Python, which is a portable version of the Cray ranf() random number generator (Dubois, 1997). Additionally, the PyTix interface to the Tix 4.0 library (Shenoy, 1996) was used as a GUI and the Pmwblt interface to the Blt 2.3 library (McFarlane, 1997) for data plotting. All the additional libraries and Python extensions are public domain software like the Python interpreter itself.
A $6 \times 86$ class $\mathrm{PC}$ system running Linux 2.0.31 was used as development platform and for most of the simulations performed in section 3. The inter-site package has also been successfully installed and used on various other Unix systems (OSF1 on DEC Alpha, Solaris 5.0 on SUN UltraSparc, Irix 6.4 on SGI Origin2000), and it should run without major complications on Mac and MS Windows systems as well.

\subsection{Simulation technique}

Simulating a model essentially means to iterate over the numerical equations that define the dynamics of each of the modeled entities while maintaining causality (see Zeigler, 1986; Fishwick, 1995 for overviews of simulation techniques). This can be done either in a concurrent fashion as in actor-based systems, where the global system dynamics are entirely determined by the exchange of messages among the model entities (Hewitt, 1977; Agha, 1986), or sequentially by imposing a global time frame on the system, as is done in the various flavors of discrete event simulation (DES) (Bratley et al., 1987; Evans, 1988; Pooch and Wall, 1993).

Concurrent systems, although attractive in their capability to mimic the concurrency of real-world processes, are difficult to implement, and error tracking is notoriously difficult; therefore, a DES framework was chosen for implementing the inter-site simulator. In short, DES simulates the dynamics of a model system as a series of state changes in the set of modeled entities mediated by 'events'. Events trigger new events and change, create, or destroy entities. In what is known as the 'event-scheduling world view' of DES, all events are stored in a global priority queue in order of their scheduling time ('time-stamp'). The simulation proceeds by de-queuing and processing the event with highest priority, i.e. the smallest timestamp. Simulation time advances asynchronously with the time-stamp of the currently processed event.

Although many of the object-oriented and individual-based models mentioned in previous sections employ a DES framework, this is rarely 


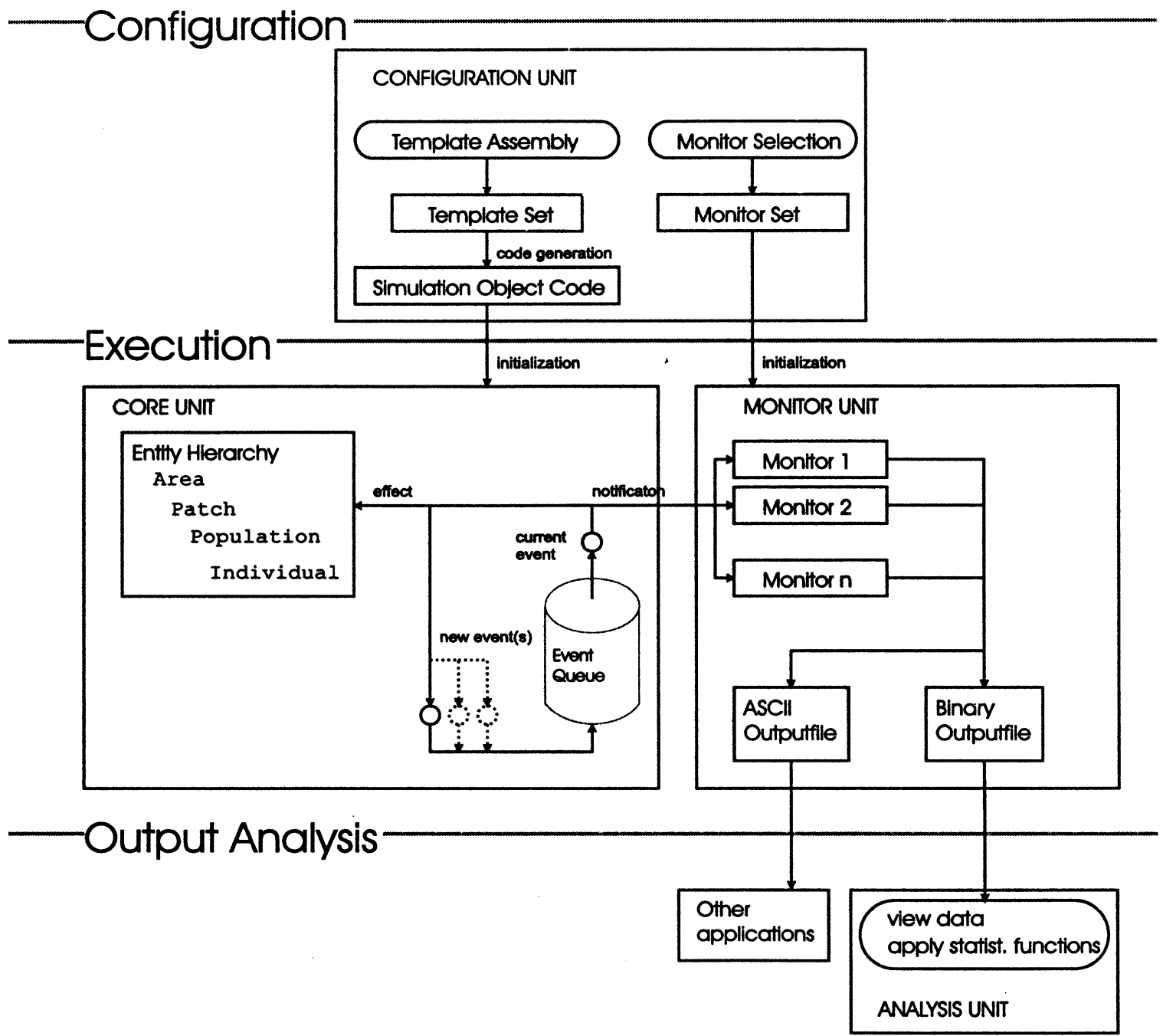

Fig. 1. Block diagram of the modular structure of the inter-site simulator

explicitly pointed out, leaving some confusion as to the details of the implementation (see Baveco and Lingeman, 1992; Hill et al., 1997 for exceptions).

Returning to our running example of modeling a meta-population system, we easily identify the DES entities as the objects introduced in section 2.1. However, the methods of the individual object classes implementing their dynamics now are separate and independent event objects, which are stored in the global event queue in order of their time-stamps.

\subsection{Architecture and basic usage}

As shown in the block diagram Fig. 1, the inter-site simulator consists of four basic functional units: the Configuration, Core, Monitor, and Analysis units. The Configuration unit contains all routines needed to assemble and parameterize the object classes subsequently used in the actual simulation process. During the simulation, the Core unit contains all instances of the simulation object classes defined during configuration. Entity object instances are collected in the 


\section{Entity Template}

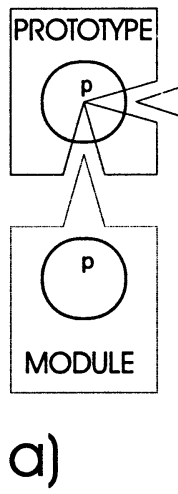

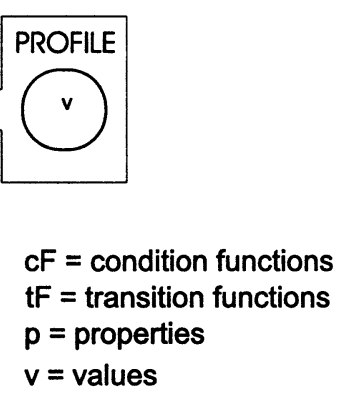

$\mathrm{CF}=$ condition functions $\mathrm{tF}=$ transition functions $p=$ properties $v=$ values

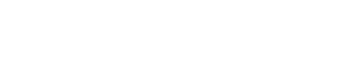

\section{Event Template}

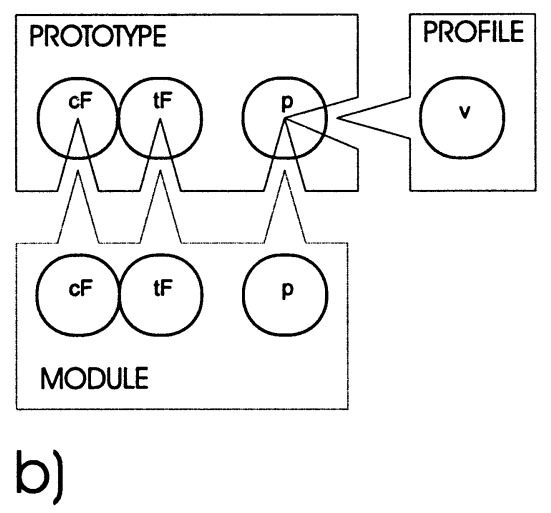

Fig. 2. Structure of Entity (a) and Event (b) Templates. Only one (optional) Module shown.

'Entity hierarchy', while event object instances are stored in the 'Event queue'. Together, these two container classes provide the routines necessary to perform the actual simulation process as it was described in the previous section. The Monitor unit contains a set of user-selected 'Monitor' objects, which are each responsible for tracking the value of a particular state variable of interest through the state changes of the entity objects in the Core unit. Finally, the Analysis unit is used for performing statistical analyses on the information gathered by the Monitor during the simulation run.

We now take a closer look at the three steps that are necessary to conduct a complete run of the inter-site simulator, configuration, execution, and analysis of the simulated model, introducing some additional terminology along the way.

\subsubsection{Configuration}

Configuring the inter-site simulator involves two steps: building a working Core and setting up monitoring.

The Core is built by assembling a set of 'Templates', a term used in OOP for classes that receive a type parameter during initialization (e.g. Pohl, 1993). Departing from this restricted definition, Templates in the inter-site simulator refer to compound class objects that serve as declarations for the classes of the actual simulation objects, which are then generated automatically (cf. Fig. 1).

A Template consists of at least two components, called its 'Prototype' and its 'Profile' (see Fig. 2). It may optionally be extended by one or several other components called 'Modules'. Assembling a Template therefore involves selecting a Prototype, a Profile, and possibly one or several Modules.

The Prototype of a Template contains the 'hard-wired' attributes and functions that are used for internal object management and that implement the intrinsic functionality of each simulation object type. Event Template Prototypes declare two additional sets of functions: a set of 'transition functions' realizing the effect of the Event (its 'action' in DES terminology) and a set of 'condition functions' specifying the potential of the Event, defined as its ability to trigger other Events. A single condition function or the logical conjunction of several condition functions of an Event may trigger zero, one, or several Events.

The Profile of a Template supplies the values of its configurable attributes, or 'Properties'. A Template may define several profiles, each containing a particular parameterization.

Finally, Template Modules serve to supplement the Prototype of either Entity or Event Templates with additional properties that require special intrinsic functionality. In addition to that, Event 


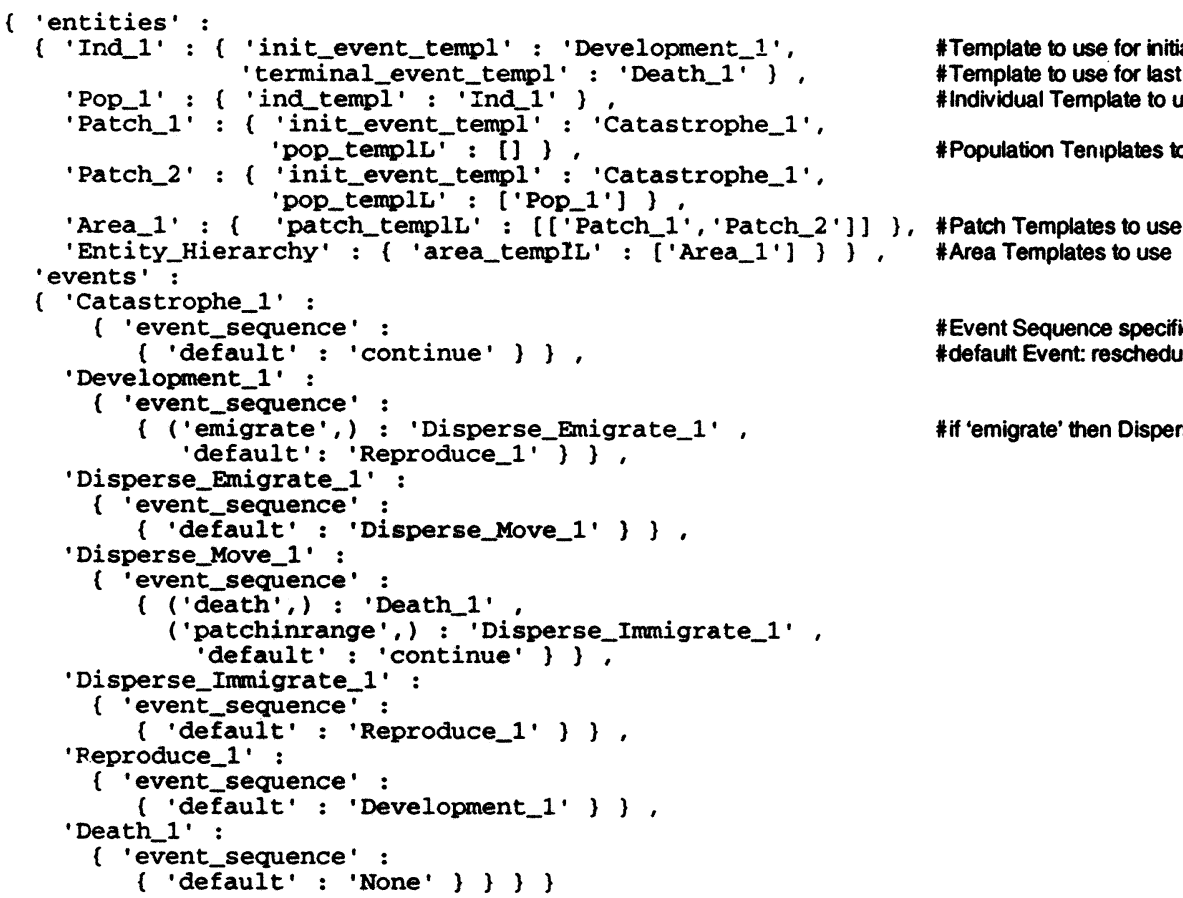

Fig. 3. The Template Set used for the sample application (Python code with comments).

Template Modules can define transition and condition functions which augment or modify the corresponding transition and condition function sets of the Prototype, respectively.

Events and Entities are linked by defining an Event Template as an initialization event of an Entity Template, i.e. as an Event to be created and scheduled during initialization of every instance of this particular Entity Template. Since most Event Templates specify at least one successor Event, specifying a single initialization Event is sufficient to subject an Entity instance to a whole sequence of dynamically created Events during runtime (the Event Sequence of the corresponding Entity).

The present implementation of the inter-site system features four Entity Template Prototypes reflecting the organizational levels of a spatially structured real-world population (Area, Patch, Population, and Individual). Among others, Event Templates implementing spatially uncorrelated environmental catastrophes for Patch-type Templates, and Event Templates implementing various development, dispersal, and reproduction modes for Individual-type Templates are available.

A set of properly assembled Entity and Event Templates that constitutes a working Core is called a Template Set (see Fig. 3 in section 3 for an example). Template Sets can be stored to a file for later reuse.

The configuration of the simulator is completed by the selection and set-up of a set of Monitor objects, each of them serving to track the value of one state variable of interest over the course of the simulation. In the inter-site system, monitoring - also called 'data collection' in DES - takes place from the stance of an omniscient, but external observer. Monitors are notified about Events happening in the Core and adjust their state accordingly; they do not depend on either Events or Entities in the Core directly. This design guarantees that Monitors will continue to work with very different Core configurations, as long as the effects of the monitored Events on the state of the Monitor remain unchanged. 
In order to get time trajectories for the state variable tracked by a Monitor object, the Monitor's state has to be periodically stored to a result data array, a process called 'snapshooting'. Monitors in the inter-site system offer three different snapshooting modes: (1) time-triggered: the Monitor state is stored in user-defined time intervals; (2) event-triggered: storing the Monitor state is triggered by a user-defined signal event, which usually indicates a particular state of the Core; and (3) continuous: each state change of the Monitor is stored.

Sets of pre-configured Monitors can be linked with a corresponding Template Set and, like the latter, stored to a file; this way, restarting the simulator in a particular configuration is just a matter of selecting the corresponding Template Set.

\subsubsection{Execution}

Execution of the simulation is done either in batch mode or under GUI control. The GUI provides routines for continuous displaying of Monitor data and inspection of Core properties. In batch mode, automated sensitivity analysis for a specified Template property with an arbitrary number of replicates is available as additional option.

\subsubsection{Analysis}

Analysis of the simulation results in the present implementation is restricted to displaying Monitor data and subjecting them to simple functions of descriptive statistics. However, routines for the hand-tailored generation of ASCII result files for data from particular Template/Monitor combinations (integrating over results from replicate runs, if necessary) are available for easy export to any spreadsheet. Additional data analysis functions are under way.

\section{A sample application: Adler and Nuernberger (1994) revisited}

\subsection{The original model}

Adler and Nuernberger (1994) present a spatially realistic state-variable model investigating the ef- fects of different patch arrangements in space on meta-population persistence. This model is ideally suited to demonstrate the capabilities of the intersite simulator because it is a simple, yet full-fledged example of a classical spatially realistic model, and because it is exceptionally well documented. To make this demonstration more interesting, we decided to use an individual-based implementation for the reconstructed model.

In their main simulation experiment, Adler and Nuernberger compare the dynamics of a meta-population system consisting of 50 patches in two different landscapes, one with a regular and the other with a clumped patch distribution in space. Local population growth follows a discrete approximation of the logistic equation with fixed values for growth rate $R$ and carrying capacity $K$ (drawn from Begon and Mortimer, 1986). During each simulation cycle, a fixed proportion $p$ of the population in each patch enters the dispersal stage, whereas the remainder of the population stays in place and reproduces. Dispersal is modeled as straight line movement across the landscape in a randomly chosen angle; to avoid border effects, periodic boundary conditions are assumed. Dispersal involves a certain mortality risk and the probability $\mathrm{a}_{\mathrm{ij}}$ that a disperser successfully travels the distance $\delta_{\mathrm{ij}}$ from patch $\mathrm{i}$ to $\mathrm{j}$ is given by:

$a_{\mathrm{ij}}=(1 / 2)^{\delta_{\mathrm{ij}} / b}$

with $b$ being the 'movement range' of the disperser, defined as the distance that can be traveled with a survival probability of 0.5 (Wilcove et al., 1986). The first patch located within a pre-set detection distance $\in$ along the movement path is selected as target patch for immigration. Successful immigration is followed by reproduction in the target patch.The only other explicit source of mortality in the model are environmental catastrophes, which are spatially uncorrelated and affect each individual patch with a fixed probability of 0.05 per simulation cycle.

In a fully factorial experiment including the three life history parameters $R, p$, and $b$, Adler and Nuernberger explore the conditions for meta-population persistence in the two landscapes. Starting with a configuration in which all patches were filled to a carrying capacity $K=200$, each parameter 
Table 1

Configurable properties of the Templates used for the sample application. Templates without configurable properties are not listed

\begin{tabular}{|c|c|c|c|c|}
\hline \multicolumn{2}{|l|}{ Template } & \multicolumn{2}{|l|}{ Properties } & \multirow[t]{2}{*}{ Value(s) } \\
\hline type & name & name & description & \\
\hline AREA & Area 1 & $\begin{array}{l}\text { size } \\
\text { pattern_option }\end{array}$ & $\begin{array}{l}\text { physical size } \\
\text { determines patch pattern }\end{array}$ & $\begin{array}{l}(200,200) \\
\text { 'regular' and 'clumped'† }\end{array}$ \\
\hline POPULATION & Pop_1 & $\begin{array}{l}\text { n_init } \\
\text { capacity } \\
\text { growth_rate }\end{array}$ & $\begin{array}{l}\text { initial } \mathrm{N} \\
\text { carrying capacity } \\
\text { growth rate }\end{array}$ & $\begin{array}{l}50 \\
100 \\
1.5,1.8\end{array}$ \\
\hline \multirow[t]{3}{*}{ EVENT } & $\begin{array}{l}\text { Development_1 } \\
\text { Disperse move }\end{array}$ & $\begin{array}{l}\text { p_emigrate } \\
\text { corridor width }\end{array}$ & $\begin{array}{l}\text { probability for emigration } \\
\text { width of patch detection corridor }\end{array}$ & $\begin{array}{l}0.03,0.25,0.5 \\
2\end{array}$ \\
\hline & -1 & & & \\
\hline & Catastrophe_1 & $\begin{array}{l}\text { move_length } \\
\text { angle } \\
\text { p_survival } \\
\text { p_occurrence }\end{array}$ & $\begin{array}{l}\text { move length } \\
\text { angle of departure } \\
\text { probability of survival } \\
\text { probability of occurrence }\end{array}$ & $\begin{array}{l}2,3,6 \\
\text { random integer in }(0,359) \\
0.5 \\
0.05\end{array}$ \\
\hline
\end{tabular}

\footnotetext{
${ }^{\dagger}$ the 'clumped' pattern was realized with a 2-level homogeneous Poisson process (Diggle 1983)

* move_length and $\mathrm{p} \_$survival constitute together the 'movement range' of the A\&N model
}

setting was run over 1000 simulation cycles and replicated 50 times. Two main observations were made: (a) persistence was generally much higher in the landscape with the clumped patch pattern; and (b) intermediate levels of dispersal resulted in the highest total meta-population size.

\subsection{The reconstruction}

Although the reconstruction of the Adler and Nuernberger model was easily possible within the framework provided by the inter-site simulator, two minor inconsistencies remained: (1) despite the otherwise extensive documentation of the original model, the values for two parametersthe size of the landscape and the width of the patch detection corridor during dispersal - were missing and had to be estimated; and (2) within our computational constraints, only 300 simulation cycles in 10 replicates with $K$ set to 100 and one quarter of the patches (at least one patch/ cluster in the clumped landscape) filled with $K / 2$ individuals as starting configuration turned out to be feasible.

Fig. 3 shows the template set and Table 1 the template property settings used for the simulations.
A virtual time unit of $1 \mathrm{~h}$ was deemed sufficiently line-grained for resolving the temporal dynamics of the processes included in the model. A simulation cycle was then defined in analogy to a real-time year, consisting of $24 \times 30 \times 12=8640$ virtual time units. The modeled organism (as specified by the Template Ind_1) was assumed to begin its life cycle in April and to reproduce for the first time, possibly following a short dispersal stage, after a developmental phase of 11 months. Every individual reiterates this cycle of development, optional dispersal, and reproduction until its virtual lifetime is terminated during Disperse Move or by a catastrophe event.

Rather than censusing the actual population size in fixed time intervals, the 'effective population size' monitor of the simulator was used for data collection. This monitor tracks the number of reproduction events per simulation cyclesummed over all local populations - and is therefore a direct measure of the number of individuals that reached the reproductive phase.

\subsection{Running the reconstruction}

Fig. 4 and Fig. 5 show the effective population size, averaged over ten replicate runs, resulting 
a) $R=1.5$, move_length $=2$

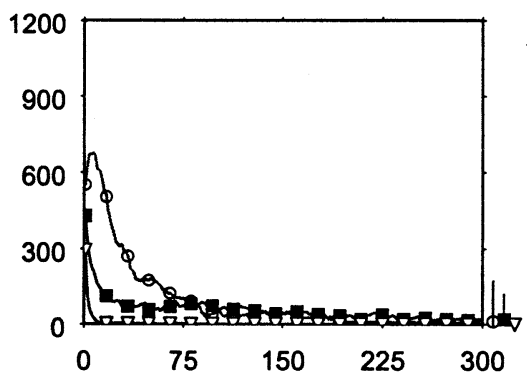

c) $R=1.5$, move_length $=3$

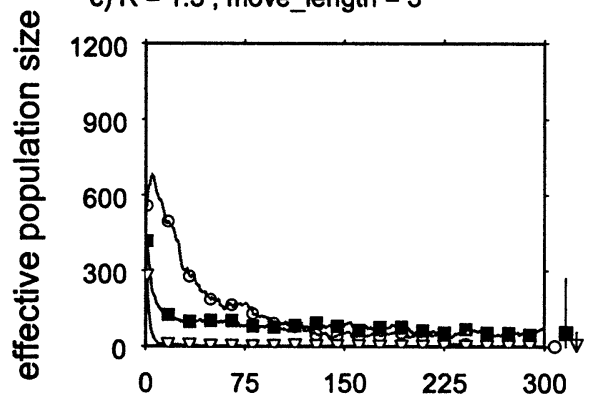

e) $R=1.5$, move_length $=6$

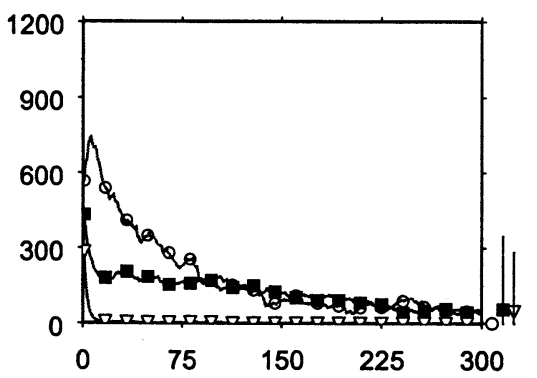

b) $R=1.8$, move_length $=2$

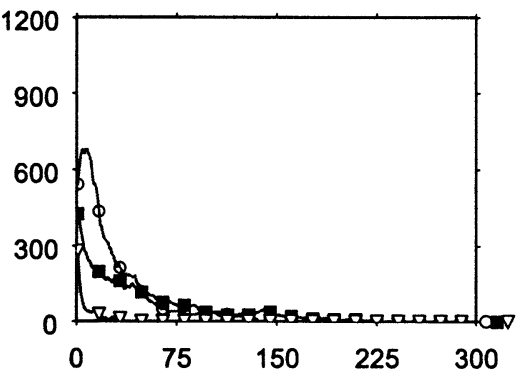

d) $R=1.8$, move_length $=3$

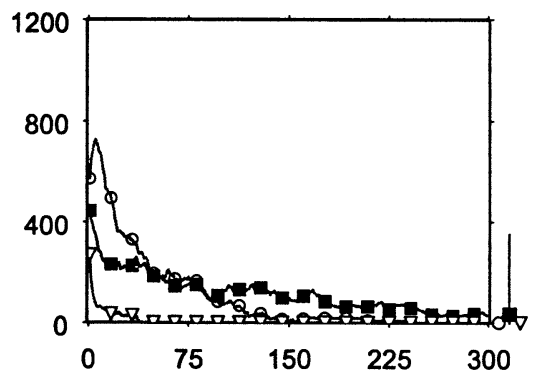

f) $R=1.8$, move_length $=6$

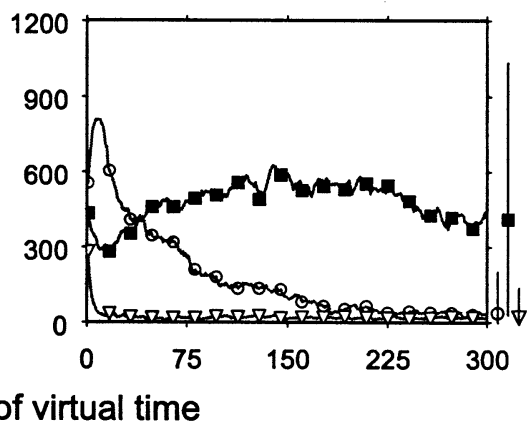

Fig. 4. Results from the regular landscape. Parameter settings in panels: columns: growth_rate $=1.5$ (left) and growth_rate $=1.8$ (right); rows: move_length $=2$ (top), move_length $=3$ (middle), move_length $=6$ (bottom). Plots in panels: each plot is averaged over ten replicate runs. $x$-axis: number of simulated generations; $y$-axis: effective population size. Circles: $p_{-}$emigrate $=0.03$; squares: $p_{-}$emigrate $=0.25$; triangles: $p_{-}$emigrate $=0.5$. Bar plots next to each panel: average (position of symbol) and extreme values (end points of bars) of all ten replicates over the last 50 generations.

from simulations varying the parameters $\mathrm{p}_{-}$emigrate (corresponding to $p$; values $0.03,0.25,0.5$ ), growth_rate (corresponding to $R$; values $1.5,1.8$ ) and move_length (corresponding to $b$; values 2, $3,6)$ in two landscapes differing in the spatial patch arrangement ('regular' and 'clumped').

The results confirm the findings of Adler and Nuernberger in that most of the parameter combi- nations for the regular patch pattern lead to rapid extinction, while the majority of the runs for the clumped patch pattern persisted for 300 generations. Also in concordance with the original model, settings with an intermediate probability of dispersal produced the highest effective population sizes, especially in combination with a high movement range in the clumped landscape. These 
a) $R=1.5$, move_length $=2$

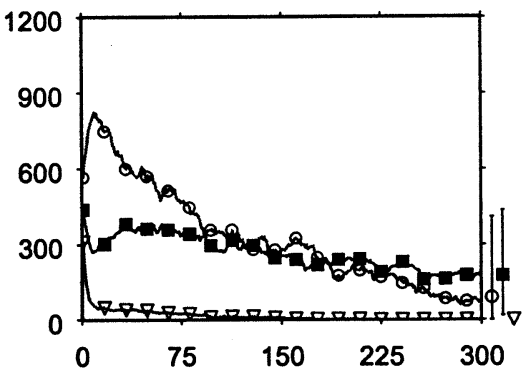

c) $R=1.5$, move_length $=3$

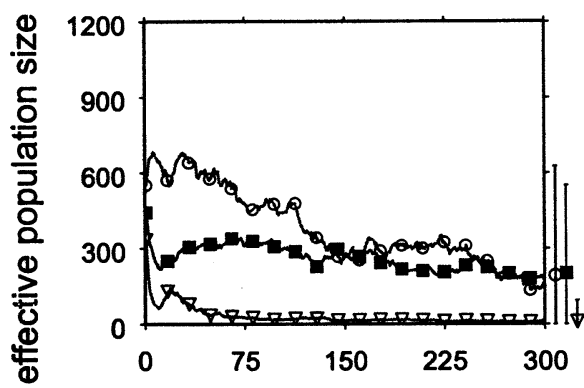

e) $R=1.5$, move_length $=6$

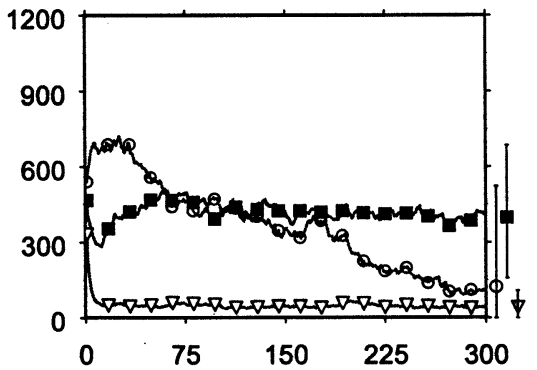

b) $R=1.8$, move length $=2$

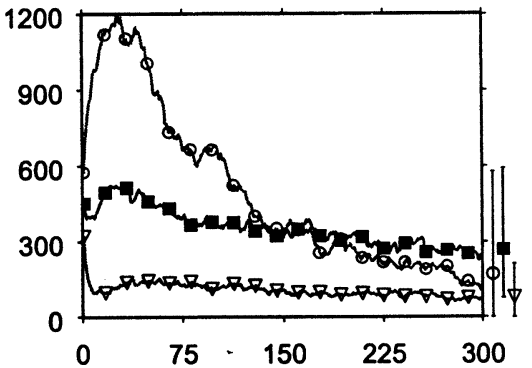

d) $R=1.8$, move_length $=3$

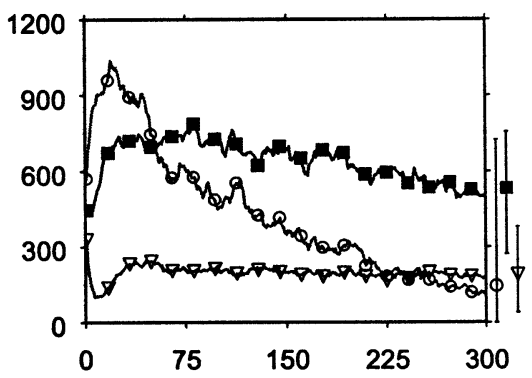

f) $R=1.8$, move_length $=6$

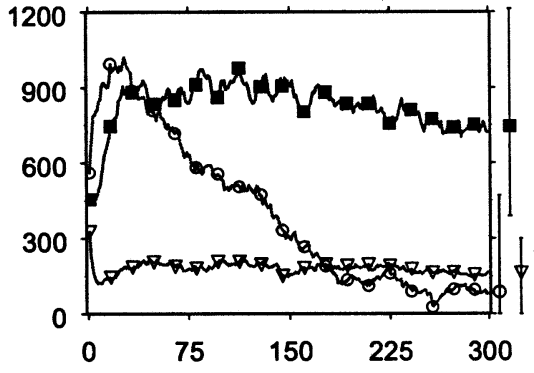

years of virtual time

Fig. 5. Results from the clumped landscape. (see caption of Fig. 4 for explanation)

results make intuitive sense, since (a) low average inter-patch distance in patch clusters facilitates the recolonization of patches affected by catastrophes; and (b) an intermediate level of dispersal should optimize its associated costs (death risk) and benefits (colonization of empty patches).

However, obtaining these particular results required considerable fine-tuning of the parameters. For example, in a smaller landscape of size
$100 \times 100$ the regular patch pattern produced higher effective population sizes than the clumped pattern for the largest movement range. Similarly, doubling the patch detection corridor width in the large landscape favored the low over the intermediate dispersal rate. Using the sensitivity analysis tool of the inter-site simulator, these and other parameter value boundaries for the 'intuitive' behavior of the system could conveniently be explored. 
Inclusion of additional data from the simulation output helped to further analyze the results obtained with the averaged effective population sizes. For instance, inspection of the absolute minimum and maximum effective population sizes over the last 50 simulation cycles (bar plots in Figs. 4 and 5) revealed that, in the clumped landscape, for most parameter combinations at least one of the ten replicate runs did not persist over 300 generations. Also, fluctuation in the effective population size was generally higher for lower dispersal rates.

In an applied context, our main goal is to manage a real-world metapopulation system in a way as to ensure that certain state variables regarded as main descriptors of the system's state stay within pre-defined limits. Here, recognition of the boundaries in parameter space delimiting the desired system behavior as well as knowledge about the dynamic range of the descriptive state variables are indispensable for deciding upon alternative management strategies.

\section{Discussion}

The results presented in the previous section introduce the inter-site simulator as a robust simulation tool that is easily adapted to capture the structure and reproduce the dynamics of a given spatial population model. Although emulating traditional state-variable models is certainly valuable for testing purposes, the main domain of application for the simulator is spatially realistic models of moderate to high complexity. However, ecologists have not yet reached a consensus about the merits of this recent breed of simulation models and the conditions under which they should be employed. Huston et al. (1988) strongly advocate the use of individual-based models, claiming that incorporating individual variability into population models leads to a more adequate description of real-world populations. On the contrary, critics argue that since individual-based models lack the mathematical rigor of their traditional counterparts, and since program code is hardly ever published, they produce somewhat arbitrary results (Judson, 1994). In a similar vein, Wenner- gren et al. (1995) warn that detailed spatially explicit or realistic models require solid parameter estimates from high-quality field data, which are hard to obtain and therefore rare. In an attempt to clarify these issues, DeAngelis and Rose (1992) provide the following criteria for appropriate use of the individual-based approach: (1) small populations sensitive to stochastic fluctuations; (2) a large number of relevant i-state variables resulting in a high degree of variation among individuals; and (3) populations in highly stochastic environments. Taking these as a guideline, it seems that individual-based models - and with them more general simulation systems like inter-site-are very likely to increase even more in popularity since (1) the results from recent large-scale field studies have begun to provide the level of detail that is necessary to support high-resolution models; and (2) increasing numbers of animal and plant species are experiencing a dramatic loss in their overall population size as well as in the average size of their local populations due to ongoing habitat destruction and fragmentation by man, rendering them highly vulnerable to stochastic fluctuations of the environment (e.g. Mangel and Tier, 1994; see also the contributions in Hanski and Gilpin, 1997).

Another objection that has been put forward against individual-based models is that they do not scale up, i.e. they are unable to provide information on higher levels than the population (de Roos and Sabelis, 1995). However, the intersite system is not affected by this limitation, since it employs a hierarchically structured approach to modeling real-world entities in a flexible DES framework rather than a strictly individual-based methodology.

The choice of a scripting language for implementing a simulation system might seem odd at first, since interpreted languages typically have a much lower execution speed than compiled languages. However, runtime performance might not be of primary concern, given the power of modern computer hardware. Moreover, the Python interpreter features a well-defined C-API and it is common practice among software programmers to benefit from the much faster development cycles of an interpreted language until the code 
stabilizes and then to move time-critical sections into an extension of the interpreter written in a compiled language for speedup. The inter-site simulator already makes extensive use of this technique and most of the Event transition functions as well as the bulk of the Core functionality has been re-implemented in $\mathrm{C}$.

Three general avenues for the future extension of the inter-site system are envisioned:

1. routines for multivariate statistical analysis of spatially structured metacommunities during simulation time ('animated ordinations'): while there is a substantial amount of literature available on single-species metapopulation systems, exploration of the dynamics of spatially structured metacommunities has only just begun (Tilman, 1994; Holt, 1997; Nee et al., 1997; Tilman et al., 1997). Combining a simulation framework with well-understood multivariate ordination techniques seems a particularly promising approach here;

2. database interfaces allowing access to species and to GIS data: crucial for the practical application of simulation models is their proper parameterization (e.g. Poethke et al., 1996; Hanski, 1997). Entering these data manually is very time-consuming even for models of moderate complexity. Structured interfaces to existing database standards would therefore markedly enhance the productivity of the inter-site system;

3. a concurrent version of the simulator: moving the simulator to a parallel architecture would be very promising in terms of performance improvements (see Haefner, 1992), but would also require in-depth changes to its internal architecture. Actor-based programming provides an appropriate framework for this task (Senteni, 1997). In fact, some of the important characteristics of actor-based programming, namely time-stamped message passing, delegation, and replacement behaviors, are already part of the present implementation of the inter-site simulator.

Given the open architecture of the simulator and its underlying programming language, Python, many other fascinating extensions are conceivable. However, the future prospect of the project depends much on how the efforts of the authors to provide a versatile and extensible simulation package are received by the ecological modelling community.

\section{Acknowledgements}

This study was supported by a HSP-II-AUFE scholarship of the DAAD, Germany, by the Schmeil Stiftung, Germany, and by the Natural Sciences and Engineering Research Council of Canada. Professor R. Hansell gave advice in the early stages of the project and J. Rennerich supplied its illuminating name. The authors would like to thank Jonathan Beecham, Ulrike Tappeiner and Richard Storey for their critical comments on earlier versions of the manuscript.

\section{References}

Adler, F.R., Nuernberger, B., 1994. Persistence in patchy irregular landscapes. Theor. Pop. Biol. 45, 41-75.

Agha, G., 1986. Actors: A Model of Concurrent Computation in Distributed Systems. MIT Press, Cambridge, MA, p. 144.

Baveco, J.M., Lingeman, R., 1992. An object-oriented tool for individual-oriented simulation: host-parasitoid system application. Ecol. Model. 61, 267-286.

Begon, M., Mortimer, M., 1986. Population Ecology: A Unified Study of Animals and Plants, 2nd ed. Blackwell Scientific, Oxford.

Blanche, S., Casas, J., Bigler, F., Janssenvanbergeijk, K.E., 1996. An individual-based model of Trichogramma foraging behavior: parameter-estimation for single females. J. Appl. Ecology 33 (3), 425-434.

Booch, G., 1994. Object-Oriented Analysis and Design with Applications. Benjamin/Cummins, Redwood City, CA, p. 589.

Bratley, P., Fox, B.L., Schrage, L.E., 1987. A Guide to Simulation. Springer, New York, p. 397.

Bullock, J., 1994. Individual-based models. Trends Ecol. Evol. 9 (8), 299.

Caswell, H., John, A.M., 1992. From the individual to the population in demographic models. In: DeAngelis, D.L., Gross, L.J. (Eds.), Individual-Based Models and Approaches in Ecology. Chapman \& Hall, New York, pp. 36-61.

Chesson, P.L., 1981. Models for spatially distributed populations: the effect of within-patch variability. Theor. Pop. Biol. 19, 288-325. 
de Roos, A.M., Sabelis, M.W., 1995. Why does space matter? In a spatial world it is hard to see the forest before the trees. Oikos 74, 347-348.

DeAngelis, D.L., Adams, S.M., Breck, J.E., Gross, L.J., 1984. A stochastic predation model: applicaton to largemouth bass observations. Ecol. Model. 24, 25-41.

DeAngelis, D.L., Rose, K.A., 1992. Which individual-based approach is most appropriate for a given problem? In: DeAngelis, D.L., Gross, L.J. (Eds.), Individual-Based Models and Approaches in Ecology. Chapman \& Hall, New York, pp. 67-87.

Diggle, P.J., 1983. Statistical Analysis of Spatial Point Patterns. Acacemic Press, London, p. 148.

Dubois, P., 1997. URL ftp://ftp-icf.llnl.gov/pub/python/RNG10.tgz.

Ermentrout, G.B., Edelstein-Keshet, L., 1993. Cellular automata approaches to biological modeling. J. Theor. Biol. $160,97-133$.

Evans, J.B., 1988. Structures of Discrete Event Simulation. Ellis Horwood, Chichester, p. 279.

Ferreira, J.G., 1995. Ecowin: an object-oriented ecological model for aquatic ecosystems. Ecol. Model. 79, 21-34.

Fishwick, P.A., 1995. Simulation model design and execution: building digital worlds. Prentice Hall, Englewood Cliffs, NJ, p. 448.

Gilpin, M.E., Hanski, I. (Eds.), 1991. Meta-population Dynamics: Empirical and Theoretical Investigations. Academic Press, London.

Graham, I., 1994. Object Oriented Methods. Addison-Wesley, Workingham, UK, p. 473.

Gross, L.J., Rose, K.A., Jr., E.J.R., Winkle, W.V., Werner, E.E., 1992. Individual-based modeling: summary of a workshop. In: DeAngelis, D.L. Gross, L.J. (Eds.), Individual-Based Models and Approaches in Ecology, Chapman \& Hall, New York, pp. 511-522.

Gustafson, E.J., Gardner, R.H., 1996. The effect of landscape heterogeneity on the probability of patch colonization. Ecology 77 (1), 94-107.

Haefner, J.W., 1992. Parallel computers and individual-based models: an overview. In: DeAngelis, D.L., Gross, L.J. (Eds.), Individual-Based Models and Approaches in Ecology. Chapman \& Hall, New York, pp. 126-164.

Hanski, I., 1994. A practical model of meta-population dynamics. J. Anim. Ecol. 63, 151-162.

Hanski, I., 1997. Metapopulation dynamics: from concepts and observations to predictive models. In: Hanski, I., Gilpin, M.E. (Eds.), Meta-population Biology: Ecology, Genetics, and Evolution. Academic Press, San Diego, pp. $69-92$.

Hanski, I., Gilpin, M.E. (Eds.), 1997. Meta-population Biology: Ecology, Genetics, and Evolution. Academic Press, San Diego, p. 512.

Hanski, I., Simberloff, D., 1997. The meta-population approach, its history, conceptual domain, and application to conservation. In: Hanski, I., Gilpin, M.E. (Eds.), Metapopulation Biology: Ecology, Genetics, and Evolution. Academic Press, San Diego, pp. 5-26.
Harrison, S., 1994. Meta-population and conservation. In: Edwards, P.J. (Ed.), Large-Scale Ecology and Conservation Biology. Blackwell, Oxford, pp. 111-128.

Harrison, S., Stahl, A., Doak, D.F., 1993. Spatial models and spotted owls: exploring some biological issues behind recent events. Conserv. Biol. 7, 950-953.

Hassell, M.P., Comins, H.N., May, R.M., 1991. Spatial structure and chaos in insect population dynamics. Nature 353, $255-258$.

Hastings, A., 1990. Spatial heterogeneity and ecological models. Ecology 71, 426-428.

Hewitt, C.E., 1977. Viewing control structures as patterns of passing messages. Artif. Intell. 8, 323-364.

Hill, D., Coquillard, P., Vaugelas, J., 1997. Discrete-event simulation of alga expansion. Simulation 68 (5), 69-277.

Holt, R.D., 1997. From meta-population dynamics to community structure: some consequences of spatial heterogeneity. In: Hanski, I., Gilpin, M.E. (Eds.), Meta-population Biology: Ecology, Genetics, and Evolution. Academic Press, San Diego, pp. 149-164.

Hugunin, J., 1997. URL http://www.sls.lcs.mit.edu/ jjh/ numpy/.

Humphries, H.C., Coffin, D.P., Lauenroth, W.K., 1996. An individual-based model of alpine plant-distributions. Ecol. Model. 84 (1-3), 99-126.

Huston, M., DeAngelis, D., Post, W., 1988. New computer models unify ecological theory. Bioscience 38, 682-691.

Jaworska, J.S., Hallam, T.G., Schultz, T.W., 1996. A community model of ciliate Tetrahymena and bacteria Escherichia coli: 1. individual-based models of Tetrahymena and Escherichia coli populations. Bull. Math. Biol. 58 (2), 247264.

Judson, O.P., 1994. The rise of the individual-based model in ecology. Trends Ecol. Evol. 9 (1), 9-14.

Kaneko, K., 1992. Overview of coupled map lattices. Chaos 2 (3), 279-282.

Kareiva, P.M., 1990. Population dynamics in spatially complex environments: theory and data. Phil. Trans. R. Soc. Lond. Ser. B 330, 175-190.

Lahaye, W.S., Gutierrez, R.J., Akçakaya, H.P., 1994. Spotted owl meta-population dynamics in Southern California. J. Anim. Ecol. 63 (4), 775-785.

Laval, P., 1995. Hierarchical object-oriented design of a concurrent, individual-based, model of a pelagic Tunicate bloom. Ecol. Model. 82, 265-276.

Levins, R., 1970. Extinction. In: Gerstenhaber, M. (Ed.), Some Mathematical Problems in Biology. American Mathematical Society, Providence, RI, pp. 75-107.

Lhotka, L., 1994. Implementation of individual-oriented models in aquatic ecology. Ecol. Model. 74, 47-62.

Liu, J.Jr., J.B.D., Pulliam, H.R., 1995. Potential effects of a forest management plan on Bachman's sparrows (Aimophila aestivalis): linking a spatially explicit model with GIS. Conserv. Biol. 9, 62-75.

MacKay, N.A., 1992. Evaluating the size effects of lampreys and their hosts: application of an individual-based model. In: DeAngelis, D.L., Gross, L.J. (Eds.), Individual-Based Models and Approaches in Ecology. Chapman \& Hall, New York, pp. 278-294. 
Maley, C.C., Caswell, H., 1993. Implementing i-state configuration models for population dynamics: an object-oriented programming approach. Ecol. Model. 67, 259-284.

Mangel, M., Tier, C., 1994. Four facts every conservation biologist should know about persistence. Ecology 75 (3), 607-614.

Maxwell, T., Costanza, R., 1997. A language for modular spatio-temporal simulation. Ecol. Model. 103, 105-113.

McFarlane, G., 1997. URL http://www.dscpl.com.au/pmw.

McKelvey, K., Noon, B.R., Lamberson, R.H., 1993. Conservation planning for species occupying fragmented landscapes: the case of the northern spotted owl. In: Kareiva, P.M., Kingslover, J.G., Huey, R.B. (Eds.), Biotic Interactions and Global Change. Sinauer, Sunderland, MA, pp. 424-450.

Metz, J.A.J., Diekmann, O. (Eds.), 1986. The Dynamics of Physiologically Structured Populations: Lecture Notes in Biomathematics, vol. 68, Springer, Berlin.

Mooij, W.M., Boersma, M., 1996. An object-oriented simulation framework for individual-based simlations (OSIRIS): Daphnia population dynamics as an example. Ecol. Model. 93, 139-153.

Morris, D.W., 1992. Earth's peeling veneer of life. Nature 373, 25.

Murdoch, W.W., McCauley, E., Nisbet, RM., Gurney, W.S.C., de Roos, A.M., 1992. Individual-based models: combinint testability and generality. In: DeAngelis, D.L., Gross, L.J. (Eds.), Individual-based models and approaches in ecology. Chapman \& Hall, New York, pp. $18-35$.

Nee, S., May, R.M., Hassell, M.P., 1997. Two-species metapopulation models. In: Hanski, I., Gilpin, M.E. (Eds.), Meta-population Biology: Ecology, Genetics, and Evolution. Academic Press, San Diego, pp. 123-148.

Palmer, J.B., 1992. Hierarchical and concurrent individual based modeling. In: DeAngelis, D.L., Gross, L.J. (Eds.), Individual-Based Models and Approaches in Ecology. Chapman \& Hall, New York, pp. 188-207.

Poethke, H.J., Gottschalk, E., Seitz, A., 1996. Gefährdungsgradanalyse einer räumlich strukturierten population der Westlichen BeiBschrecke (Platycleis albopunctata): Ein Beispiel fur den Einsatz des Metapopulationskonzeptes im Artenschutz (German with English abstract).
Pohl, I., 1993. Object-Oriented Programming Using $\mathrm{C}++$. Benjamin Cummings, Redwood City, CA, p. 496.

Pooch, U.W., Wall, J.A., 1993. Discrete Event Simulation. A Practical Approach. CRC Press, Boca Raton, FL, p. 412.

Reich, M., Grimm, V., 1996. Das metapopultionskonzept in ökologie und naturschutz: eine kritische bestandsaufnahme (German with English abstract). Z. Ökol. Naturschutz 5 (3-4), 123-139.

Rose, K.A., Tyler, J.A., Chambers, R.C., Kleinmacphee, G., Danila, D.J., 1996. Simulating winter flounder populationdynamics using coupled individual-based young-of-theyear and age-structured adult models. Can. J. Fish. Aquat. Sci. 53 (5), 1071-1091.

Senteni, A., 1997. Steps to an ecology of actor simulation. In: Zobrist, G.W., Leonard, J.V. (Eds.), Object-Oriented Simulation: Reusability, Adaptability, Maintainability. IEEE Press, New York, pp. 269-318.

Shenoy, S., 1996. URL http://www2.gol.com/users/sshenoy/ PyTix.html.

Stelter, C., Reich, M., Grimm, V., Wissel, C., 1994. Ein modell zur dynamik einer metapopulation von Bryoderna tubemulata (Saltatoria, Acrididae): kann diese art überleben? (German with English abstract). Verh. Ges. Ökol. 23, 383-390.

Tilman, D., 1994. Competition and biodiversity in spatially structured habitats. Ecology 75 (1), 2-16.

Tilman, D., Lehman, C.L., Yin, C., 1997. Habitat destruction, dispersal, and deterministic extinction in competitive communities. Amer. Nat. 149 (3), 407-435.

Uchmanski, J., Grimm, V., 1996. Individual-based modeling in ecology: what makes the difference? Trends Ecol. Evol. 11 (10), 437-441.

van Rossum, G., 1997. URL http://www.python.org/doc/.

Wennergren, U., Ruckelshaus, M., Kareiva, P., 1995. The promise and limitations of spatial models in conservation biology. Oikos 74, 349-356.

Wilcove, D.S., McLellan, C.H., Dobson, A.P., 1986. Habitat fragmentation in the temperate zone. In: S.M. (Ed.), Conservation Biology: The Science of Scarcity and Diversity, Sinauer Ass., Sunderland, MA, pp. 237-256.

Zeigler, B.P., 1986. Toward a simulation methodology for variable structure modeling. In: Elzas, M.S., Ören, T.I., Zeigler, B.P. (Eds.), Modeling and Simulation Methodology in the Artificial Intelligence Era. Elsevier, Amsterdam, pp. 195-210. 